\title{
Fragment and schema models for recall
}

\author{
GREGORY V. JONES \\ University of Bristol, Bristol, England
}

\begin{abstract}
Two general conceptualizations of recall may be distinguished. One holds that recall is subserved by memory traces in which representations of different aspects of external events are linked directly to each other. The other holds that these representations are connected via a mediating concept. Formalizations of these views are provided by two theories examined by Ross and Bower (1981a): the "fragment" and "schema" models, respectively. Ross and Bower found that patterns of cued recall of clusters of words with a common theme were predicted well by the schema model, but not by the fragment model. Two experiments reported here show that the reverse is true if the clusters of words are selected randomly. Ross and Bower also considered separately a third theory, the "horizontal" model. However, the horizontal model is shown here to be a constrained form of the fragment model. Another constrained form of the fragment model, termed the "link" model, is introduced and is shown to provide an account of encoding as well as of retrieval that, although it is outstandingly parsimonious, is also approximately accurate. Finally, it is shown that the memory structures characterized by the fragment model may be interpreted as either graphs or digraphs: that is, networks with either bidirectional or unidirectional connections between nodes.
\end{abstract}

The question addressed here is that of how best to characterize the building blocks of the recall process. In particular, consider the memory representation that corresponds to an event with several different aspects. How are the different components corresponding to these aspects linked in memory? Two types of answer to this question have been offered, distinguishing the two dominant schools of memory inquiry during the last half century. The older school, derived from philosophical associationism (see Anderson \& Bower, 1973), holds that the different components are linked directly to one another. But the newer school, that of constructivism, originating with Bartlett (1932), holds that the components are grouped by virtue of their common connections to some higher order structure.

One way in which to attempt to discriminate between these two types of view is by means of cued recall. In recent years, two different theoretical models-one within each of the schools--have both enjoyed some success in quantitatively predicting the results of experiments of this type. These theories are the "fragment" model (Jones, 1976, 1978c; Jones \& Payne, 1982) and the "schema" model (Ross \& Bower, 1981a; see also Anderson \& Bower, 1973). For ease of exposition, these models are outlined in the following sections in the context of the type of task used in both the present experiments and those of Ross and Bower (1981a).

In the experimental task, subjects initially study clusters of four nouns. Subsequently, they receive either one or two words of each cluster as retrieval cues. As a

I am grateful to an anonymous reviewer for helpf ul suggestions about exposition. Requests for reprints should be sent to Gregory V. Jones, Department of Psychology, University of Bristol, 8-10 Berkeley Square, Bristol BS8 1HH, England. result, the probabilities of occurrence of seven different pattems of recall may be estimated empirically. These are zero, one, two, or three words recalled with a oneword cue, and zero, one, or two words recalled with a two-word cue. A group of patterns of recall of approximately this size has two useful properties. On the ont hand, it is sufficiently large to allow differentiation of the two models under discussion. On the other hand, it is sufficiently compact for enough data to be gathered concerning the incidence of each pattern to allow the models to be applied to individual subjects. The frag. ment and schema models to be compared are describec next.

\section{Fragment Model}

The fragment model is concerned with the functiona relations between cues and information that is recalled As such, it is essentially abstract in form. But it is con venient to introduce it in terms of a specific interpreta tion as a form of memory network model, rather than a: a mathematical abstraction. For the case of a four-wor cluster, this is illustrated by Figure 1.

According to the model, the encoding of each cluste consists of a set of nodes (each corresponding to a word directly connected by links. On any particular occasion the structure encoded will be either the complete on shown in Figure 1 or else some smaller part of it. When : word is provided as a cue, it retrieves those other word (if any) to which there are intact links. If two words an provided as a cue, they similarly retrieve any words tt which links from one or the other (or both) are intact According to this scheme, five different levels of com pleteness of encoding can be distinguished, and ar termed five different types of fragment. 


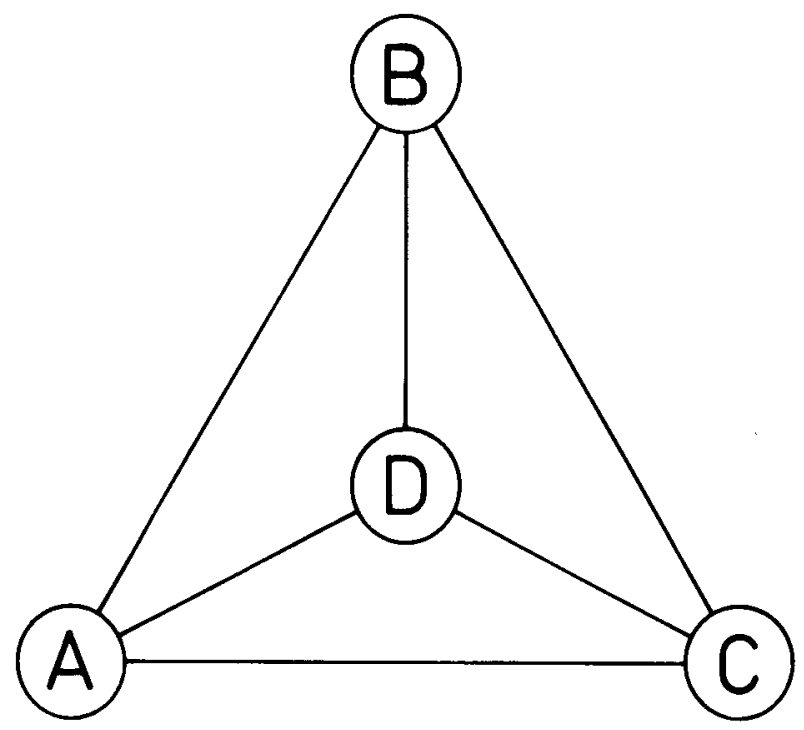

Figure 1. Representation of the fragment model's memory structure for a word quartet, with internode links depicted as the six edges of a tetrahedron.

In a complete fragment, termed Type (4), all four nodes are linked. In a Type $(3,1)$ fragment, three nodes are linked. In a Type $(2,1,1)$ fragment, two nodes are linked. In a Type $(2,2)$ fragment, all four nodes are linked, but as two separate pairs. Finally, in a Type $(1,1,1,1)$ fragment, none of the nodes are linked. For example, after studying the quartet of words $\mathrm{A}, \mathrm{B}, \mathrm{C}$, and D, a person might retain a Type $(2,2)$ fragment in which $A$ is linked to C and B is linked to D. Then, each of the four possible types of one-word cue would lead to the recall of a single word (e.g., D would produce B). On the other hand, one-third of the six two-word cues (e.g., B with D) would produce no recall, whereas the remaining twothirds would each lead to the recall of two words (e.g., $B$ with $C$ would produce $A$ and D). The explication in a similar way of the patterns of recall expected for each of the other combinations of cue and of fragment enables one to predict the overall disposition of responses, as follows.

Let the probability of occurrence of a fragment of Type (i) be denoted by $f_{(i)}$, and the probability of recalling $n$ words when cued by $m$ words be denoted by $P(n \mid m)$. Then, the expected probabilities of recall are:

$$
\begin{aligned}
& P(0 \mid 1)=(1 / 4) f_{(3,1)}+(1 / 2) f_{(2,1,1)}+f_{(1,1,1,1)} \\
& P(1 \mid 1)=(1 / 2) f_{(2,1,1)}+f_{(2,2)} \\
& P(2 \mid 1)=(3 / 4) f_{(3,1)} \\
& P(3 / 1)=f_{(4)} \\
& P(0 \mid 2)=(1 / 3) f_{(2,1,1)}+(1 / 3) f_{(2,2)}+f_{(1,1,1,1)} \\
& P(1 \mid 2)=(1 / 2) f_{(3,1)}+(2 / 3) f_{(2,1,1)}
\end{aligned}
$$

$$
P(2 \mid 2)=f_{(4)}+(1 / 2) f_{(3,1)}+(2 / 3) f_{(2,2)}
$$

The values of the fragment parameters, $f_{(i)}$, can themselves be obtained in several different theoretically interesting ways. Each way can be specified as a particular version of the fragment model. Four versions are outlined here: "link," "node," "horizontal," and "general" models. The fragment parameters that they specify will be referred to as $\mathrm{fL}_{(i)}, f^{N_{(i)}}, \mathrm{fH}_{(\mathrm{i})}$, and $\mathrm{fG}_{(\mathrm{i})}$, respectively. The link and node models are extremely parsimonious one-parameter theories, formulated here for the first time. The horizontal model is a two-parameter theory that may be viewed as the conjunction of the link and node models. It was formulated originally by Ross and Bower (1981a), who tested, in all, three models: horizontal, fragment, and schema. However, they did not note that, in fact, the first and second are different members of the same family of theories, treated here as the horizontal and general versions, respectively, of the fragment model. The general version is the form in which the fragment model has usually been tested previously (e.g., Jones, 1976, 1978c; Jones \& Payne, 1982). The four different versions are described next.

Link model. According to the link model, the different types of fragment result from the failure to encode different combinations of the internode links shown in
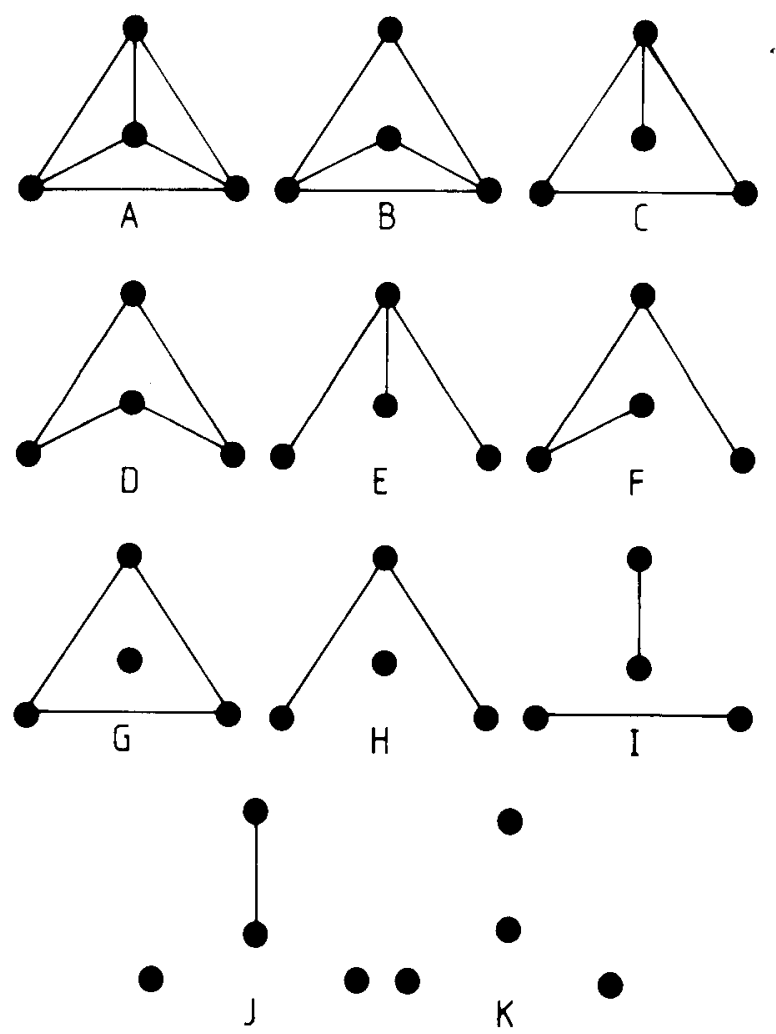

Figure 2. Representations of the 11 memory structures distinguished by the link version of the fragment model. Only encoded links are depicted. 
Figure 1. It is assumed that each of the links is encoded independently, and hence it is possible to predict the pattern of fragments on the basis of a single parameter expressing the probability of encoding of each link.

In the four-component case, there are six links between nodes, and thus 64 (i.e., 26 ) distinct combinations of encoded and unencoded links. These combinations may be categorized first in terms of the number of encoded links for each combination. Because of the independence assumption, the distribution of these categories will be binomial. Let $\theta$ denote the probability of a link's being encoded. Then, for example, the total probability of the 15 combinations in which exactly two links are encoded is $15 \theta^{2}(1-\theta)^{4}$. There is not a one-to-one relation between binomial category and fragment type, however. The two-link case, for example, contributes 12 combinations of the type (A-B-C, D) and three of the type (A-B, C-D), corresponding to Type $(3,1)$ and Type $(2,2)$ fragments, respectively. These are shown as Structures $H$ and I, respectively, in Figure 2. Altogether, the 11 different types of nonisomorphic structure shown in Figure 2 can be distinguished (1 six-link, 1 five-link, 2 four-link, 3 three-link, 2 twolink, 1 one-link, and 1 zero-link), each one corresponding to a particular fragment type. ${ }^{1}$ Type (4) and Type $(3,1)$ fragments derive from 6 and 2 of these structures, respectively, whereas Types $(2,1,1),(2,2)$, and $(1,1,1,1)$ derive from 1 each.

The first 11 rows of Table 1 correspond to the 11 different nonisomorphic structures depicted in Figure 2. Each row lists the relevant fragment type, the number of isomorphic combinations included within that structure, and-in the link column-the predicted theoretical probability of occurrence of the structure. Grouping of these probabilities over the five types of fragment allows the prediction of the overall fragment probabilities, as follows.

For convenience of notation, let

$$
s_{j}=\theta j(1-\theta)^{6-j} \text { for } j=0,6 \text {. }
$$

Then it can be seen from Table 1 that

$$
\begin{aligned}
& \mathrm{fL}_{(4)}=\mathrm{s}_{6}+6 \mathrm{~s}_{5}+15 \mathrm{~s}_{4}+16 \mathrm{~s}_{3} \\
& \mathrm{fL}_{(3,1)}=4 \mathrm{~s}_{3}+12 \mathrm{~s}_{2} \\
& \mathrm{fL}_{(2,1,1)}=6 \mathrm{~s}_{1} \\
& \mathrm{fL}_{(2,2)}=3 \mathrm{~s}_{2} \\
& \mathrm{fL}_{(1,1,1,1)}=\mathrm{s}_{0} .
\end{aligned}
$$

As an example, the probabilities of fragments of Types (4), $(3,1),(2,1,1),(2,2)$, and $(1,1,1,1)$ for $\theta=1 / 2$ are approximately $.59, .25, .09, .05$, and .02 , respectively.

Node model. According to the node model, failures of encoding of internode links are not independent of each other (as assumed by the link model). Instead, the failures occur in groups of three links, all attached to the same node. It is convenient to alternatively describe each such group of three link failures as a failure to encode the node at which the links intersect. The node model

\begin{tabular}{|c|c|c|c|c|c|}
\hline \multirow[b]{2}{*}{ Structure* } & \multirow{2}{*}{$\begin{array}{c}\text { Fragment } \\
\text { Type }\end{array}$} & \multirow{2}{*}{$\begin{array}{l}\text { Number of } \\
\text { Isomorphs** }\end{array}$} & \multicolumn{3}{|c|}{ Theoretical Probability } \\
\hline & & & Link† & Node $t_{\dagger}$ & Horizontal \\
\hline $\mathbf{A}$ & (4) & 1 & $\theta^{6}$ & $\mathrm{t}_{4}$ & $\theta^{6} t_{4}$ \\
\hline $\mathrm{B}$ & (4) & 6 & $6 \theta^{5}(1-\theta)$ & $4_{4}$ & $6 \theta^{5}(1-\theta) \mathrm{t}_{4}$ \\
\hline $\mathrm{C}$ & (4) & 12 & $12 \theta^{4}(1-\theta)^{2}$ & & $12 \theta^{4}(1-\theta)^{2} \mathrm{t}_{4}$ \\
\hline $\mathrm{D}$ & (4) & 3 & $3 \theta^{4}(1-\theta)^{2}$ & & $3 \theta^{4}(1-\theta)^{2} t_{4}$ \\
\hline $\mathrm{E}$ & (4) & 4 & $4 \theta^{3}(1-\theta)^{3}$ & & $4 \theta^{3}(1-\theta)^{3} t_{4}$ \\
\hline $\mathrm{F}$ & (4) & 12 & $12 \theta^{3}(1-\theta)^{3}$ & & $12 \theta^{3}(1-\theta)^{3} \mathrm{t}_{4}$ \\
\hline G & $(3,1)$ & 4 & $4 \theta^{3}(1-\theta)^{3}$ & & $4 \theta^{3}(1-\theta)^{3} t_{4}$ \\
\hline $\mathrm{H}$ & $(3,1)$ & 12 & $12 \theta^{2}(1-\theta)^{4}$ & & $12 \theta^{2}(1-\theta)^{4} t_{4}$ \\
\hline I & $(2,2)$ & 3 & $3 \theta^{2}(1-\theta)^{4}$ & & $3 \theta^{2}(1-\theta)^{4} t_{4}$ \\
\hline J & $(2,1,1)$ & 6 & $6 \theta(1-\theta)^{5}$ & & $6 \theta(1-\theta)^{5} \mathrm{t}_{4}$ \\
\hline $\mathrm{K}$ & $(1,1,1,1)$ & 1 & $(1-\theta)^{6}$ & & $(1-\theta)^{6} t_{4}$ \\
\hline $\mathbf{L}$ & $(3,1)$ & 1 & & $t_{3}$ & $\theta^{3} t_{3}$ \\
\hline $\mathbf{M}$ & $(2,1,1)$ & 1 & & $t_{2}$ & $\theta \mathrm{t}_{2}$ \\
\hline $\mathbf{N}$ & $(1,1,1,1)$ & 1 & & $\mathrm{t}_{1}$ & $t_{1}$ \\
\hline 0 & $(1,1,1,1)$ & 1 & & $t_{0}$ & $t_{0}$ \\
\hline $\mathbf{P}$ & $(3,1)$ & 3 & & & $3 \theta^{2}(1-\theta) \mathrm{t}_{3}$ \\
\hline$Q$ & $(2,1,1)$ & 3 & & & $3 \theta(1-\theta)^{2} t_{3}$ \\
\hline $\mathrm{R}$ & $(1,1,1,1)$ & 1 & & & $(1-\theta)^{3} t_{3}$ \\
\hline $\mathbf{S}$ & $(1,1,1,1)$ & 1 & & & $(1-\theta) \mathrm{t}_{2}$ \\
\hline
\end{tabular}
asserts that the different types of fragment result from independent failures of this type. Figure 3 shows the five distinct types of structure allowed by the model. Each

Table 1

Memory Structure Probabilities Predicted by the Link, Node, and Horizontal Versions of the Fragment Model

*Figures 2, 3, and 4 depict Structures $A$ to $K, A$ and $L$ to $O$, and $P$ to $S$, respectively. * *Isomorphs are different combinations with the same pattern of link loss. TParameter $\theta$ represents the probability of each link's being intact. TfParameter ${ }_{k} k$ represents the probability of $k$ nodes' being intact; Equation 14 gives $t_{k}$ as a function of $p$, the probability of each node's being intact. 


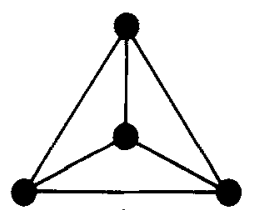

A

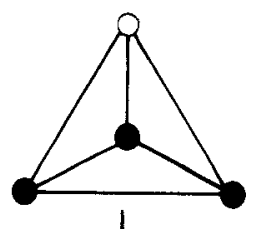

L

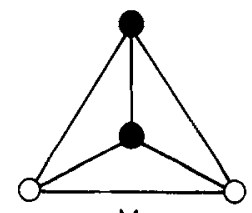

M

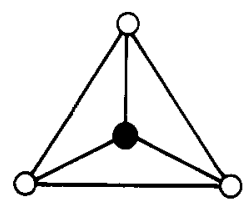

N

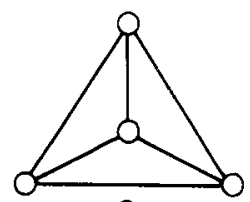

0
Figure 3. Representations of the five memory structures distinguished by the node version of the fragment model. Encoded nodes are shown as solid circles and nonencoded nodes as hollow sircles.

failure to encode a node is represented in Figure 3 by a hollow circle. Alternatively, it could be represented as deletion of the three links attached to each such node. Thus, it can be seen that Structures L, M, N, and O in Figure 3 are equivalent to Structures $\mathrm{G}, \mathrm{J}, \mathrm{K}$, and $\mathrm{K}$ (again), respectively, in Figure 2.

The derivation of fragment probabilities is simpler for the node model than for the link model, because each binomial category-that is, each number of nodes encoded-corresponds to a single type of fragment. The predicted theoretical probability of occurrence of each structure is shown as the appropriate entry in the node column of Table 1 . Let $p$ denote the probability of encoding of each node. For convenience of notation, define also

$$
t_{k}=\left(\begin{array}{l}
4 \\
k
\end{array}\right) p^{k}(1-p)^{4-k} \text { for } k=0,4
$$

where

$$
\left(\begin{array}{l}
4 \\
k
\end{array}\right)=4 ! /[k !(4-k) !]
$$

Then it can be seen from Table 1 that

$$
\begin{aligned}
& \mathrm{fN}_{(4)}=\mathrm{t}_{4} \\
& \mathrm{fN}_{(3,1)}=\mathrm{t}_{3} \\
& \mathrm{fN}_{(2,1,1)}=\mathrm{t}_{2} \\
& \mathrm{fN}_{(2,2)}=0 \\
& \mathrm{fN}_{(1,1,1,1)}=\mathrm{t}_{1}+\mathrm{t}_{0} .
\end{aligned}
$$

A noteworthy aspect of the node model is that it presludes the formation of the Type $(2,2)$ fragment. As an illustration, for $p=1 / 2$ the probabilities of fragments of Types $(4),(3,1),(2,1,1),(2,2)$, and $(1,1,1,1)$ are .06 , $.25, .38,0$, and .31 , respectively.
Horizontal model. According to the horizontal model, the different types of fragment result from independent failures of encoding both of individual links and of individual nodes (as in the node model, the failure of encoding of an individual node may alternatively be described as the failure of encoding of each of the three attached links). The horizontal model is thus a twoparameter generalization of the link and node models, characterized by the probabilities of encoding an individual link, $\theta$, and an individual node, $\mathrm{p}$.

According to the horizontal model, each of the methods of formation of memory structures allowed by the link and node models (see Figures 2 and 3, respectively) can be distinguished, together with the four further types shown in Figure 4. First, consider the structures shown in Figure 2. These correspond to complete node encodings together with link encodings of varying degree. Thus, their theoretical probabilities are shown in the horizontal column of the first 11 rows of Table 1 as the products of $t_{4}$ (the probability of complete node encoding-see Equation 14) and of the relevant link probabilities. Second, consider the structures shown in Figure 3. Here, both node and link encodings occur to varying degrees (although with the link encoding in each case occurring to the maximum level allowed by the degree of node encoding). As an example, Structure $L$ represents the successful encoding of three of the nodes, together with all three of the links between these nodes. Its probability of occurrence is therefore shown in Table 1 as the product of $t_{3}$ and of $\theta^{3}$. Finally, consider the structures shown in Figure 4. In these, both node and link encodings again occur to varying degrees, with in this case incomplete encoding both of the nodes and of the possible number of links. As an example, Structure $P$ represents the successful encoding of three of the nodes, together with that of two of the three links between these nodes. Since the latter combination may occur in three different ways, the probability of occurrence is shown in Table 1 as

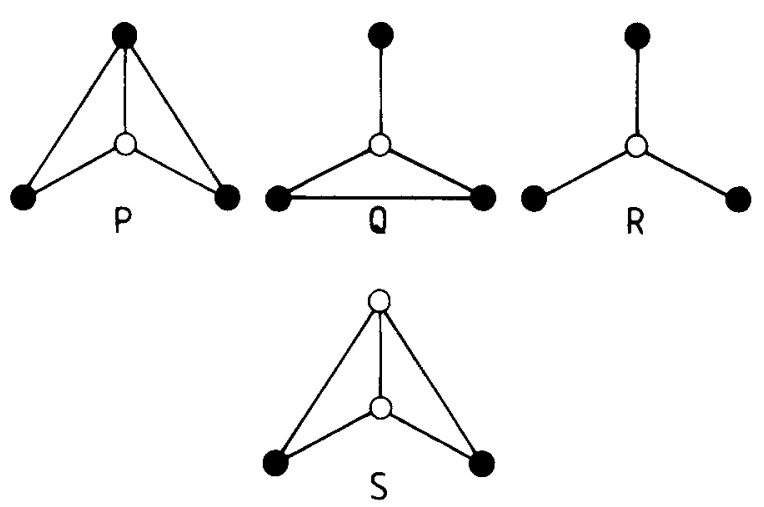

Figure 4. Representations of the four ways in which the horizontal version of the fragment model allows memory structures to arise by joint failures of encoding of links (shown blank) and of nodes (shown hollow). In addition, the horizontal model allows structures to arise by link failure alone (see Figure 2) or by node failure alone (see Figure 3). 
the product of $t_{3}$ and of $3 \theta^{2}(1-\theta)$. Because failure to encode a node may alternatively be represented as deletion of the three links attached to each such node, it may be noted that Structures $P, Q, R$, and S in Figure 4 are equivalent to Structures $\mathrm{H}, \mathrm{J}, \mathrm{K}$, and $\mathrm{K}$ (again) in Figure 2.

Grouping of all the horizontal values in Table 1 over the five types of fragment yields the overall predictions of fragment probabilities shown next. As before, expressions in $\theta^{6}$ are denoted in terms of the transform $\mathrm{s}_{\mathrm{j}}$ (Equation 8), and all expressions in $\mathrm{p}$ are denoted in terms of the transform $t_{k}$ (Equation 14).

$$
\begin{aligned}
& \mathrm{fH}_{(4)}=\left(\mathrm{s}_{6}+6 \mathrm{~s}_{5}+15 \mathrm{~s}_{4}+16 \mathrm{~s}_{3}\right) \mathrm{t}_{4} \\
& \mathrm{fH}_{(3,1)}=\left(4 \mathrm{~s}_{3}+12 \mathrm{~s}_{2}\right) \mathrm{t}_{4}+\left[\theta^{3}+3 \theta^{2}(1-\theta)\right] \mathrm{t}_{3} \\
& \mathrm{fH}_{(2,1,1)}=6 \mathrm{~s}_{1} \mathrm{t}_{4}+3 \theta(1-\theta)^{2} \mathrm{t}_{3}+\theta \mathrm{t}_{2} \\
& \mathrm{fH}_{(2,2)}=3 \mathrm{~s}_{2} \mathrm{t}_{4} \\
& \mathrm{fH}_{(1,1,1,1)}=\mathrm{s}_{0} \mathrm{t}_{4}+(1-\theta)^{3} \mathrm{t}_{3}+(1-\theta) \mathrm{t}_{2}+\mathrm{t}_{1}+\mathrm{t}_{0} .
\end{aligned}
$$

The explicit formulation of this set of equations allows one to check that both the link and the node models are special cases of the horizontal model. If $\mathrm{p}$ equals $1, t_{4}$ equals 1 and all other $t_{k} s$ equal 0 , yielding the link model. Similarly, if $\theta$ equals $1, s_{6}$ equals 1 and all other $\mathrm{s}_{\mathrm{j}} \mathrm{s}$ equal 0 , yielding the node model. In addition, the above formulation points to an important advance in the theoretical interpretation of the horizontal model originated by Ross and Bower (1981a).

In their article, Ross and Bower (1981a) compared three different models for the structure of recall. Their schema model and fragment model (corresponding to the general version of the fragment model, to be described next) constitute independent alternatives. But they did not notice that their third model, which they named the horizontal model, is not in fact an independent alternative. This was because the way in which they extracted its predictions did not display the fact that, as shown by the equations here, the horizontal model is a form of fragment model, and in particular a constrained form of the general version with which they contrasted it. ${ }^{2}$ Thus, in discussing the horizontal model, Ross and Bower commented at one point that " 10 of the 15 subjects had $\theta$ estimates of 1.00 , which effectively reduces their model to a constrained fragment model" (p. 9). The demonstration that the horizontal model is in fact itself a fragment model considerably simplifies the taxonomy of models for the structure of recall that was proposed by Ross and Bower, reducing their trichotomy to the dichotomy of fragment and schema models studied here.

General model. In the general version of the fragment model, the probabilities of the different types of fragment are each estimated independently, subject only to the constraint that they should sum to unity. Thus, in the four-word case considered here, the five $f_{(i)}$ probabilities are specified by four independent parameters; each of these parameters is equal to a fragment probability, with the fifth fragment probability being obtained by subtraction from unity. It may be noted that the general model also can be viewed as a kind of Gestalt model that does not distinguish between different structures such as the six Type (4) fragment structures, $\mathrm{A}$ to $\mathrm{F}$, shown in Figure 2.

The link, node, horizontal, and general versions of the fragment model have in common that their predictions are mediated via the values taken for the five fragment probabilities, $f_{(i)}$ (see Equations 1 to 7 ). The general version, with four independent parameters, is an unrestricted form in that it allows all possible combinations of values of the five fragment probabilities to occur (subject only to the bounds $0 \leqslant f_{i} \leqslant 1$ ). Each of the other three versions is a restricted form in that the values that may be taken by the five fragment probabilities are subject to particular constraints. ${ }^{3}$ The unconstrained nature of the general version expresses mathematically the absence of any of the restrictive psychological assumptions about fragment distributions that are made by the other versions. For example, the general version, unlike the remainder, does not assume that processing is necessarily equally distributed over the different components of a stimulus. If, however, the general version's advantage in accuracy over one of the constrained versions proved empirically to be slight, then it would be appropriate to prefer the more parsimonious version. ${ }^{4}$ It is in the form of the general version that the fragment model has usually been tested previously (e.g., Jones, 1976, 1978c; Ross \& Bower, 1981a).

\section{Schema Model}

In its qualitative form, the schema model can be traced to Bartlett (1932). But the form in which it has been advanced as a quantitative model for recall was characterized by Ross and Bower (1981a), and is illustrated (again, for a four-word cluster) by Figure 5 . The critical way in which the schema model differs from the fragment model is in its proposal that nodes corresponding to individual components are not linked to each other directly. Rather, they are linked only via an organizing schema (S in Figure 5), whose construction is a function both of previous experience and of the present encoding process.

Schema-based recall can be represented by two parameters, a and $r$. Parameter a denotes the probability that the presentation of a word as a retrieval cue will allow access to the schema to occur. Parameter $r$ denotes the probability that, following activation of the schema, a particular word will be responded. The access and response processes are assumed to be both independent of each other and also to be independent for different words. Predicted probabilities for different patterns of recall with different numbers of cues can be obtained readily For example, a complete failure of recall of a four. 


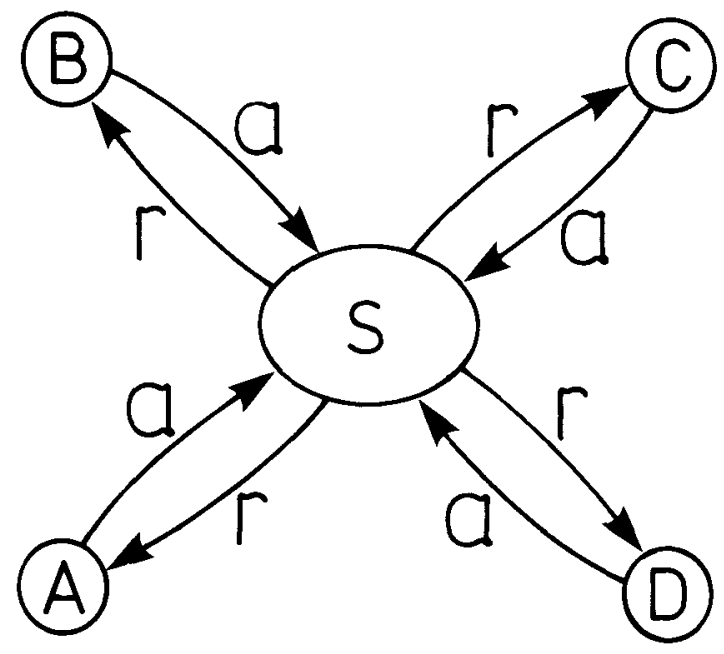

Figure 5. Representation of the schema model's memory itructure for a word quartet.

word cluster with a one-word cue arises either when the zue fails to access the schema, with probability $(1-a)$, or when it does access the schema but none of the three correct responses can be made, with probability a $(1-\mathrm{r})^{3}$. Thus, corresponding to Equations 1 to 7 for the fragment model, we have

$P(0 \mid 1)=1-a+a(1-r)^{3}$

$P(n \mid 1)=a\left({ }_{n}^{3}\right) r^{n}(1-r)^{3-n}$, for $n=1,2,3$

$P(0 \mid 2)=(1-a)^{2}+[a+a(1-a)](1-r)^{2}$

$\mathrm{P}(\mathrm{n} \mid 2)$

$$
=[a+a(1-a)]\left({ }_{n}^{2}\right) r^{n}(1-r)^{2-n}, \text { for } n=1,2 .(28
$$

In view of the fact that the horizontal model proposed by Ross and Bower (1981a) has been shown here to be a constrained form of the fragment model, it is desirable to establish that (in accord with its qualitatively different appearance) the schema model is not also another constrained form. This is demonstrated in the Appendix, where it is shown that the schema model allows the occurrence of patterns of recall other than those allowed by the general version of the fragment model.

\section{Comparison of the Models}

At present, little empirical evidence is available concerning the relative appropriatenesses of the fragment and schema models for the structure of recall, with the exception of that reported by Ross and Bower (1981a). In two experiments, Ross and Bower examined the effects of providing different numbers of words as retrieval cues for stimuli composed of four or five related words (a third experiment is discussed later in this article). In their first experiment, they found that the schema model fit the data well and that the horizontal (fragment) model was also satisfactory, but that the (general) fragment model did not fare well. Direct chi-square comparisons are possible only when one model is a constrained form of another, which has now been shown to be the case for the latter two models. Relative to the horizontal model, an increase of 32 degrees of freedom for the general model reduced Pearson's chi-square statistic by only 22.23 , indicating that the adoption of its greater generality was not justified. In Ross and Bower's second experiment, the schema model was again satisfactory, whereas both other models performed very poorly (their fits cannot be compared directly because they were applied to different configurations of data in that experiment).

The results of Ross and Bower's (1981a) second experiment show that the fragment model does not provide a satisfactory account of recall for all types of material, and in particular that in certain circumstances the representation afforded by the schema model is better. It is possible, nevertheless, that in other circumstances the reverse is true. That is, for some types of material, the fragment model may, in general, represent recall better than does the schema model. Previous work (Jones, 1976, 1978c) had suggested that the fragment model is successful in representing the recall of material with unrelated components. Consequently, in the experiments to be reported here, participants attempted to recall clusters of words that had not been selected on the basis of their preexisting relations to each other.

\section{EXPERIMENT 1}

In its design, Experiment 1 resembled that used by Ross and Bower (1981a), except in one respect. In the two experiments discussed earlier, Ross and Bower's participants were presented with clusters of words selected on individual themes (for example, in their second experiment, the experimenter always announced "red" when presenting the following cluster: ketchup, brick, robin, strawberry, firetruck). But in the present experiment, the words for each cluster were selected at random.

\section{Method}

Subjects. The 15 subjects were psychology undergraduates of Bristol University who participated in partial fulfillment of a course requirement.

Stimuli. There were eight lists of 10 items each, each item (or "quartet") consisting of four words. The overall set of 320 words was selected from those words tabulated by Paivio, Yuille, and Madigan (1969) that have imagery values greater than or equal to 5.00 and frequencies of occurrence (Thorndike \& Lorge, 1944) greater than or equal to 10 per million. Each word in the set was allocated randomly to a particular list and quartet.

Cues. Following its presentation to a subject, each quartet was subsequently cued by either one or two of its constituent words. The set of cues that each of the 15 subjects received for each list was unique, but always consisted of six one-word cues 
and four two-word cues (the proportion of one-word cues was made higher than that of two-word cues because the resulting data were to be grouped into a larger number of patterns of recall for one-word cues). The identities of the cues for each item were balanced across subjects. For each quartet, four different one-word cues and six different two-word cues are possible. The balancing ensured that, over subjects and for each quartet, three of the four possible one-word cues occurred on two occasions and one on three, and each of the six possible two-word cues occurred on exactly one occasion.

Procedure. The stimuli of each list were presented on 10 successive slides, projected onto a screen by a Kodak Carousel S-AV linked to an automatic timer. The exposure duration was $10 \mathrm{sec}$. On each slide, the four words of a quartet were typed, leftjustified, in a column.

For recall, each subject had a booklet with a separate page for each list. The page was covered with a cardboard mask containing a slit. After studying a quartet list, the subject slid the mask down the page one line at a time, exposing (in a $1.5 \mathrm{x}$ $16 \mathrm{~cm}$ slit) the successive word cues. Recalled words were written to the right of the cues. A signal to move the mask down was given every $20 \mathrm{sec}$. The purpose of the mask was to help to isolate the recall of each quartet, since the subject could not see other cues or responses. The order in which the quartets of each list were cued varied randomly for each list and subject, with the constraint that the first and second halves of each list were tested by the first and second halves, respectively, of the sequence of cues. Both halves of the sequence of cues contained three one-word cues and two two-word cues.

\section{Results}

Table 2 shows the observed frequencies of each of the possible patterns of recall for one-word and two-word cues. Also shown are the theoretical frequencies predicted by the different versions of the fragment model and by the schema model. These are considered separately for each theory. In each case, the optimal theoretical fit was obtained using the NAG routine E04JAF (Numerical Algorithms Group, 1981) to minimize the Pearson chi-square statistic, with upper and lower boundary values of zero and one, respectively, for each theory parameter (in the case of the four-parameter general version of the fragment model, for each of the five fragment probabilities). All models were fit to the data for each subject individually, and the theoretical frequencies shown in Table 2 represent in each case the sum of the frequencies for individual subjects. Similarly, all mean parameter values cited in the following were obtained by averaging values obtained for individual subjects.
Link fragment model. The mean value of the param eter $\theta$ of the link model was .354 . The mean values 0 : the five fragment parameters, $\mathrm{fL}_{(\mathrm{i})}$, derived from the $\theta$ parameters are shown in Table 3 (as usual, averaged oves subjects). The comparison of data and theory has foul degrees of freedom for each subject, since there is ont parameter to be estimated and the seven data categorie: are subject to constraints on the totals of one-cue anc two-cue data. Overall, the data adhered quite well to the form predicted by the model, although the test statisti reached significance $\left[\chi^{2}(60)=91.81, .005<p<.01\right]$ Individually, the fits for three subjects were discrepan at the .05 level.

Node fragment model. The mean value of the param eter $p$ of the node model was .696 . The resulting mear values of the five fragment parameters, $\mathrm{fN}_{(\mathrm{i})}$, are show in Table 3 . The data adhered poorly to the form pre dicted by the model $\left[\chi^{2}(60)=179.46, p<.001\right]$ Individually, seven subjects' fits were discrepant at thi .05 level.

Horizontal fragment model. The mean values of thi parameters $\theta$ and $\mathrm{p}$ of the horizontal model were .47 : and .886 , respectively. The resulting mean values of th five fragment parameters, $\mathrm{fH}_{(\mathrm{i})}$, are shown in Table 3 The comparison of data and theory has three degrees o freedom for each subject. The data did not adhere wel to the form predicted by the model $\left[\chi^{2}(45)=84.11\right.$ $\mathrm{p}<.001]$. Individually, five subjects' fits were discrep ant at the .05 level.

General fragment model. The mean values of the fiv fragment parameters, $f_{(i)}$, are shown in Table 3. Thi comparison of data and theory has one degree of free dom for each subject. Overall, the data agreed wel with the model's predictions $\left[\chi^{2}(15)=20.28, p>.1\right]$ with only one subject's fit descrepant at the .05 level

Comparing the different versions of the fragmen model, it is apparent from the foregoing that the genera model is the most adequate version, even when allow ance is made for its higher number of parameters, bu that the link model also predicts the results' configura tion relatively successfully. Comparison of the goodness of-fit statistics confirms this view. The horizontal mode accounts for little more variation than the link mode $\left[\chi^{2}(15)=7.70, p>9\right]$, but considerably more than th node model $\left[\chi^{2}(15)=95.35, p<.001\right]$. The genera

Table 2

Experiment 1 Empirical and Theoretical Frequencies

\begin{tabular}{lccccccc}
\hline & \multicolumn{4}{c}{ One-Cued Recall } & & \multicolumn{3}{c}{ Two-Cued Recall } \\
\cline { 2 - 5 } \cline { 5 - 7 } Frequencies & 0 & 1 & 2 & 3 & & 0 & 1 \\
\hline Empirical & 224 & 132 & 104 & 260 & 115 & 120 & 245 \\
Link Fragment & 217.9 & 125.3 & 136.7 & 240.1 & 104.0 & 138.2 & 237.8 \\
Node Fragment & 237.8 & 86.4 & 186.7 & 209.1 & 97.8 & 159.8 & 222.4 \\
Horizontal Fragment & 228.7 & 107.1 & 140.5 & 243.7 & 108.6 & 135.3 & 236.1 \\
General Fragment & 222.7 & 133.1 & 103.2 & 261.0 & 119.6 & 117.0 & 243.4 \\
Schema & 231.5 & 91.3 & 183.0 & 214.2 & 99.0 & 156.5 & 224.5 \\
\hline
\end{tabular}

Note-Theoretical frequencies are the sums of predictions for individual subjects. 
Table 3

Experiment 1 Fragment Frequencies (in Percent) for Different Models

\begin{tabular}{lccccc} 
& \multicolumn{5}{c}{ Fragment Type } \\
\cline { 2 - 6 } \multicolumn{1}{c}{ Model } & $(4)$ & $(3,1)$ & $(2,1,1)$ & $(2,2)$ & $(1,1,1,1)$ \\
\hline Link & 33.4 & 25.3 & 24.2 & 5.3 & 11.8 \\
Node & 29.0 & 34.6 & 24.0 & 0 & 12.4 \\
Horizontal & 33.9 & 26.0 & 22.8 & 3.5 & 13.9 \\
General & 36.2 & 19.1 & 22.2 & 7.4 & 15.0 \\
\hline
\end{tabular}

Note-The percentages are the means of values for individual subjects.

model accounts rather better for the data than does the link model $\left[\chi^{2}(45)=71.53, .005<p<.01\right]$ and accounts much better than does the node model $\left[\chi^{2}(45)=\right.$ $159.18, p<.001]$ or the horizontal model $\left[\chi^{2}(30)=\right.$ $63.83, \mathrm{p}<.001]$.

Schema model. The mean values of the parameters $\mathrm{a}$ and $\mathrm{r}$ of the schema model were .709 and .698 , respectively. The comparison of data and theory has three degrees of freedom for each subject. Overall, the data adhered poorly to the form predicted $\left[\chi^{2}(45)=131.35\right.$, $\mathrm{p}<.001]$. Individually, seven subjects' fits were discrepant at the .05 level.

\section{Discussion}

The results of Experiment 1 were in good agreement with the fragment model, but not with the schema model. Indeed, the accuracy of the two-parameter schema model was considerably less than that of the one-parameter link version of the fragment model. Furthermore, as can be seen from Table 2, the fit of the schema model was not merely quantitatively poor but was also qualitatively poor, with the predicted frequency of recalling two words to one cue, for example, being nearly twice the observed value. It may in fact be readily deduced from Equation 26 that, according to the schema model, a constant relation should hold among the probabilities of recalling one, two, or three words to one cue. This relation takes the form

$$
\mathrm{P}(2 \mid 1)=[3 \mathrm{P}(1 \mid 1) \mathrm{P}(3 \mid 1)]^{1 / 2},
$$

since by substitution we have the identity

$$
3 \operatorname{ar}^{2}(1-r)=\left[3.3 \operatorname{ar}(1-r)^{2} \cdot \operatorname{ar}^{3}\right]^{1 / 2} .
$$

Detailed examination of the data revealed, however, that for every single subject the observed frequency of recalling two words to one cue was less than the value predicted by Equation 29 .

Considering now the different versions of the fragment model, the configuration of results was predicted surprisingly well by the extremely parsimonious link model. However, the best model for these results was provided by the general version of the fragment model.
Examination of Table 3 suggests that the advantage of the general over the link model may have derived from its allowance of greater levels of complete success and complete failure in encoding [i.e., of Type (4) and Type $(1,1,1,1)$ fragments, respectively]. This deviation from the predictions of the link model may have been a consequence of some variability in item difficulty. Encoding of each of the links (see Figure 1) could have occurred independently for each stimulus (as asserted by the link model), but with a varying level of success over stimuli. The result would be an apparent excess of both complete success and complete failure in encoding.

Levels of recall in Experiment 1 were relatively high, particularly in the two-cue case. There has been some suggestion that level of performance is a salient factor in experiments of this type (Foss \& Harwood, 1975; Jones, 1978c). Consequently, a second experiment, in which it was aimed to achieve a lower level of recall overall, was carried out.

\section{EXPERIMENT 2}

Experiment 1 followed the procedure of Ross and Bower (1981a) in its choice of exposure duration for each stimulus. In Experiment 2, the exposure duration was halved in order to lower the level of subsequent recall.

\section{Method}

There were 15 new subjects from the same source as those in Experiment 1.

In other respects, the method of this experiment resembled that of Experiment 1, except that each slide was exposed at presentation for $5 \mathrm{sec}$ instead of $10 \mathrm{sec}$.

\section{Results}

The results of Experiment 2 were analyzed similarly to those of Experiment 1. Table 4 shows the observed and theoretical frequencies, summed over subjects. The fits of the individual models were as follows.

Link fragment model. The mean value of the parameter $\theta$ was .269; the mean values of the five fragment parameters, $\mathrm{fL}_{(\mathrm{i})}$, derived from $\theta$ are shown in Table 5 . Overall, the agreement between data and model (shown in Table 4) was quite good, although the test statistic reached significance $\left[\chi^{2}(60)=86.54, .01<p<.05\right]$. Individually, the fits for four subjects were discrepant at the .05 level.

Node fragment model. The mean value of the parameter $\mathrm{p}$ was .604; the values of $\mathrm{f}^{\mathrm{N}}$ (i) are shown in Table 5. Overall, the agreement between data and model was poor $\left[\chi^{2}(60)=146.34, p<.001\right]$. Individually, the fits for seven subjects were discrepant at the .05 level.

Horizontal fragment model. The mean values of the parameters $\theta$ and $\mathrm{p}$ were .471 and .829 , respectively; the corresponding values of $\mathrm{fH}_{(i)}$ are shown in Table 5 . Overall, the agreement between data and model was 
Table 4

Experiment 2 Empirical and Theoretical Frequencies

\begin{tabular}{lccccccc}
\hline & \multicolumn{4}{c}{ One-Cued Recall } & & \multicolumn{3}{c}{ Two-Cued Recall } \\
\cline { 3 - 5 } \multicolumn{1}{c}{ Frequencies } & 0 & 1 & 2 & 3 & 0 & 1 & 1 \\
\hline Empirical & 298 & 151 & 119 & 152 & 178 & 126 & 176 \\
Link Fragment & 295.0 & 152.1 & 133.9 & 138.9 & 150.5 & 160.0 & 169.5 \\
Node Fragment & 314.6 & 110.0 & 175.8 & 119.6 & 146.3 & 175.9 & 157.9 \\
Horizontal Fragment & 311.3 & 126.5 & 141.7 & 140.5 & 157.1 & 157.0 & 165.8 \\
General Fragment & 305.5 & 149.8 & 116.3 & 148.4 & 175.5 & 123.0 & 181.5 \\
Schema & 313.5 & 114.6 & 169.2 & 122.6 & 150.4 & 170.3 & 159.2 \\
\hline
\end{tabular}

Note-Theoretical frequencies are the sums of predictions for individual subjects.

Table 5

Experiment 2 Fragment Frequencies (in Percent) for Different Models

\begin{tabular}{lccccc}
\hline & \multicolumn{5}{c}{ Fragment Type } \\
\cline { 2 - 6 } Model & $(4)$ & $(3,1)$ & $(2,1,1)$ & $(2,2)$ & $(1,1,1,1)$ \\
\hline Link & 19.3 & 24.8 & 31.4 & 5.4 & 19.1 \\
Node & 16.6 & 32.5 & 30.6 & 0 & 20.3 \\
Horizontal & 19.5 & 26.2 & 29.4 & 2.9 & 22.0 \\
General & 20.6 & 21.5 & 22.3 & 9.7 & 25.9 \\
\hline
\end{tabular}

Note-The percentages are the means of values for individual subjects.

quite good, although the test statistic reached significance $\left[\chi^{2}(45)=70.05, .005<p<.01\right]$. Individually, the fits for two subjects were discrepant at the .05 level.

General fragment model. The mean values of the five fragment parameters, $\mathrm{fG}_{(\mathrm{i})}$, are shown in Table 5 . Overall, the data agreed well with the model's predictions $\left[\chi^{2}(15)=15.98, \mathrm{p}>.3\right]$, with no individual subject's fit being discrepant.

Comparing the different forms of fragment model, the general model is thus the most adequate version, but the link and horizontal models are also quite successful. Quantitatively, the horizontal model was no better than the link model $\left[\chi^{2}(15)=16.49, p>.3\right]$, but was considerably better than the node model $\left[\chi^{2}(15)=\right.$ $76.29, p<.001]$. The general model accounted for the data rather better than the link model $\left[\chi^{2}(45)=70.56\right.$, $.005<\mathrm{p}<.01]$ or the horizontal model $\left[\chi^{2}(30)=\right.$ $54.07, .001<\mathrm{p}<.005]$ and much better than the node model $\left[\chi^{2}(45)=130.36, p<.001\right]$.

Schema model. The mean values of the parameters a and $r$ were .623 and .606 , respectively. Overall, there was poor agreement between data and model $\left[\chi^{2}(45)=\right.$ $99.52, \mathrm{p}<.001]$. Individually, four subjects' fits were discrepant at the .05 level.

\section{Discussion}

The mean levels of recall in this experiment $(39.1 \%$ and $49.8 \%$ for single-cued and double-cued recall, respectively) were, as expected, considerably lower than those in Experiment 1 (51.9\% and 63.5\%, respectively). But, in spite of this, the results provided evidence closely consistent with the conclusions drawn from those of the previous experiment. The fit of the schema model, for example, was again qualitatively as well as quantitatively poor (see Table 4), and, again, its accuracy was worse than that of the link model in spite of its extra parameter. Examination of the posited relation given by Equation 29 showed that, in Experiment 2, the observed frequency of recalling two words to one cue was less than the value predicted by the schema model for all except two subjects. The fragment model, on the other hand, was again successful in predicting the patterns of recall. As before, the best model for the results was provided by its general version. However, the link model again proved to provide a useful approximation, as before differing principally in its expectation of slightly lower levels of all-or-none encoding (and also of twinfragment formation).

\section{GENERAL DISCUSSION}

The predictions of two different types of model for the structure of recall were tested in the experiments reported here. There has been some variation in the previous formulation of both fragment models (e.g., Broadbent, 1981; Le Voi \& Rawles, 1979; Richardson, 1983; Wilhite, 1982) and, in particular, schema models (e.g., Abelson, 1981; Anderson, Kline, \& Beasley, 1979; Rumelhart \& Ortony, 1977). But a consistent point of contrast is discemible, and was focused on by the two formal models that were tested. The fragment model posits that the representations of different aspects of an event are directly linked to each other in memory, whereas the schema model specifies that links between components are not direct but, instead, are mediated by a further, central structure. The results of both experiments unambiguously supported the fragment over the schema model. The general relation between the two types of model is considered next, followed by discussion of the successful fragment model alone.

\section{Direct and Indirect Recall}

The implications of the present study are most usefully viewed in juxtaposition with those of Ross and Bower (1981a). Ross and Bower reported the results of experiments that were similar to the present ones ex. cept in using sets of related rather than unrelated words, 
nd that supported the schema rather than the fragment nodel. Taken together, the results of the two studies uggest, therefore, the existence of two distinct types of ecall. Indirect recall is favored when cues and targets rear a preexisting relation to each other, and direct ecall is favored when they do not (see also Jones, 983). Note, however, that the distinction would be xpected to be only approximately realized for any iven material. On the one hand, a preexisting relation mong words may be ignored or overlooked, with encodng then occurring via the direct links used for unrelated vords. On the other hand, nominally unrelated words re in fact to some degree related, and thus are prone to ndirect linkage. For example, it has been shown that the nembers of up to approximately $10 \%$ of pairs of ranlomly selected words are judged to exhibit strong senantic relations to each other (Ellis \& Marshall, 1978).

I have previously reviewed evidence for a dualnechanism view of recall (Jones, 1978a, 1980), but dentified then the indirect route with one of generaion followed by recognition (c.g., Bahrick, 1970, 1979). lowever, the schema model has the advantage of empirial support for its greater generality of application in particular, to multiply as well as singly cued recall). iurthermore, it appears also to have the potential to mbrace the generation-recognition model as a special ase. At retrieval, generation could be identified with the ccessing of a schema together with a listing of its conents, followed by a recognition decision upon each of hese. Thus, on this account, the schema model's reponse parameter, $r$, would represent the product of oth listing and recognition. One simple corollary is hat the value of $r$ should be less than or equal to the orresponding recognition probability. As yet, however, lata bearing on this point are not available.

Both the fragment and schema models have been lescribed here in terms of localized memory traces with graph-like or network internal structure. However, it hould be noted that these descriptions serve only to llustrate in concrete form relations between cues and argets in recall that are specified in more general, bstract form by the relevant equations. It is the apropriateness of the latter, rather than of the former, hat is directly tested empirically. Thus, in principle, a letwork structure may alternatively be denoted by a eature representation (e.g., Hollan, 1975). Similarly, roperties of localized memory traces can be simulated y distributed memory systems (e.g., Hinton, 1981). 'or example, the fragment model's assumption that a etrieval cue redintegrates the entire trace with which it verlaps could be instantiated by a simple content. ddressable distributed memory system that employs onvolution and correlation as input and output operaions, respectively (see Murdock, 1979).

A degree of flexibility is possible also in the way that he relation between the fragment and the schema rodels is construed. It was pointed out earlier that these two models may be taken as being characteristic of two different general views of memory: the associationist and the constructivist traditions, respectively. The framework utilized by Ross and Bower (1981a) in comparing the horizontal and schema models suggests, however, an alternative interpretation. According to this reasoning, the fragment and schema models are both concerned with a process of trace construction, and differ only in terms of the quantitative values that they posit for a single common factor, the span of intersection. According to this view, the encoding of any cluster in memory involves the connection of its different components via mediating concepts. These mediating concepts are located as points of intersection of activation spreading from each component, as detailed, for example, in the ACT model of Anderson (1976). The schema model represents the case of maximum overlap in intersection: All the paths between components intersect at a common point. The fragment model represents the converse case of minimum overlap: The point of intersection is unique for each pair of components. For the four-word clusters investigated here, for example, the schema model posits a single point of intersection with a span of four, whereas the fragment model posits six points of intersection, each with a span of two. The theoretical integration of schema and fragment models offered by the intersection-span approach is attractive. What distinguishes it from the alternative view is the additional postulate that the simple binary links of the fragment model are formed, not by direct, but instead, by indirect, mediation. However, it may prove difficult in practice to obtain unambiguous evidence for this postulate.

Finally, in view of its success in the experiments reported here, the fragment model is considered more fully next.

\section{Fragment Model}

Four different forms of the fragment model were tested here. They share the basic characteristic of positing that all the members of the particular set of components (i.e., fragment) that go to make up an individual memory become available for recall when one (or more) of their number overlaps with a retrieval cue. But, in contrast with this common characterization of retrieval, the four forms differ in their further assumptions about encoding. The link and the node models postulate that the basic units of encoding are the link between components and the component itself, respectively; the horizontal model postulates that both these types of encoding unit are relevant; and the general model does not specify patterns of fragment formation. Thus, the link. node, and horizontal models are theories of both encoding and retrieval, whereas the general model is one of retrieval alone. Of these different versions of the fragment model, it was the general one to which the data fitted best. However, there was also considerable consis- 
tency with the link model, and it is informative in several respects to consider this further.

As previously discussed, the appropriateness of the fragment model as a whole is a function of the type of material whose recall is studied. In the present experiments, the material consisted of clusters of randomly selected high-frequency words. Retrospectively, it thus made sense that there should be particular support for the link model, since this implies that the basic determinant of the patterns of storage was the difficulty of encoding relations between pairs of words, and not that of encoding the words themselves. More generally, the parsimony of the account of patterns of encoding that is offered by the link model makes it a useful tool in understanding this aspect of memory in more qualitative terms.

Consider, for example, the formation of multiple fragments-two or more traces derived from the same stimulus, independently giving rise to recall with appropriate cues. Since the classic experiments of Anderson and Bower (1973, chap. 10; see also Jones, 1978c), it has consistently been found that estimates of the frequency of occurrence of multiple fragments have been very low (of the order of a few percent). Now, the existence of multiple independent traces is a postulate that has no counterpart in the schema model, and thus it was argued by Ross and Bower (1981a) that the fact that estimates of their occurrence are in practice relatively close to zero is tantamount to evidence against the fragment notion as a whole. The link model, however, enables this argument to be refuted, since it provides a principled explanation of the phenomenon. The only type of multiple fragment in the present experiments is the Type $(2,2)$ trace, and its frequency of occurrence is predicted by the link model to be always relatively low. As for other multiple fragments, this is because it is yielded by very few configurations of links, each of which requires the numbers of links encoded successfully and unsuccessfully to be roughly comparable. We may readily obtain the maximum expected probability of the Type $(2,2)$ fragment by differentiating the expression in Equation 12 with respect to the link probability $(\theta)$, setting the result to zero, and solving. This yields a value for $\theta$ of $1 / 3$, corresponding to a maximum probability of occurrence of only $16 / 243$, or about $7 \%$. Although small in magnitude, the contribution of multiple fragments is probably quite important. It is noteworthy that the node model, the only version of the fragment model to disallow multiple fragments, fared much the worst empirically.

The link model also provides a convenient vehicle for outlining an alternative interpretation of the fragment parameters. Hitherto, they have been regarded as the probabilities of different patterns of encoding. But an alternative is that encoding is complete at presentation, and that the fragment parameters represent the consequences of subsequent forgetting. That is, rather than $\theta$ 's representing the probability of forming a link, $(1-\theta)$ represents the probability of forgetting a link. Similarly, differences in estimated values of $\theta$ over subjects would result from differences in the speed, not of encoding, but instead, of forgetting. For each person, initial complete fragments would eventually degrade into null fragments, but via the creation and subsequent destruction of partial fragments. The Type $(2,2)$ fragment was noted earlier to occur maximally when the link forgetting probability would be equivalent to $2 / 3$, and, similarly, it can be shown that maximal occurrences of the Type $(2,1,1)$ and Type $(3,1)$ fragments (Equations 11 and 10, respectively) would occur if the forgetting probability were $5 / 6$ and $(1+\sqrt{17}) / 8$ (approximately .64 ), respectively. Note, however, that any interpretation of the fragment probabilities must also account for the fact that increasing the speed of stimulus presentation in Experiment 2 resulted in levels of recall generally lower than those in Experiment 1. A forgetting interpretation of fragment probabilities would have to attribute this phenomenon to an increase in the fragility of links when these are formed under speeded encoding conditions.

\section{Graphical or Digraphical Memories?}

Finally, we turn to a point of considerable generality the question of whether the links between nodes in : memory representation are bidirectional or unidirec tional. This may be expressed as the question of whethe memory traces correspond to graphs or to digraph: (where a digraph is a directed graph-see Wilson, 1972 chap. 7), and is more subtle than the question of whethes or not overall associative symmetry prevails (cf. Ascr \& Ebenholtz, 1962). Thus far, the fragment and schem: models have been taken as corresponding to graph anc digraph representations, respectively: For Figures 1 to $\angle$ (illustrating the fragment model), all links were inter preted as bidirectional, whereas for Figure 5 (illustratin! the schema model), each link has separate access an response components in opposite directions. However the results of experiments in which each memory $i$ cued only once (e.g., those reported here) do not allov one to exclude a digraph interpretation of the fragmen model that is precisely equivalent to the graph inter pretation.

According to both the graph and digraph interpreta tions, a fragment parameter represents the probabilit: of occurrence of a set of one or more related types $o$ memory trace. The set of possible digraphs is in genera larger than the set of graphs, because each link in th compiete graph structure is replaced in the correspond ing digraph structure by a pair of directional links o "arcs." Figure 6 illustrates the simple case of tw different nodes, for which there are two distinct graph and four distinct digraphs.

Digraph analogues of graph interpretations of frą ment parameters can be constructed in at least tw different ways. In both, each digraph arc occurs with th same probability as the corresponding graph link. I 
GRAPHS

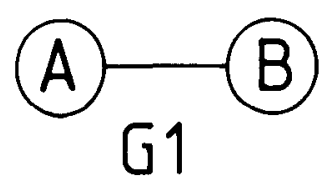

(A)

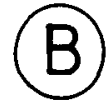

G2
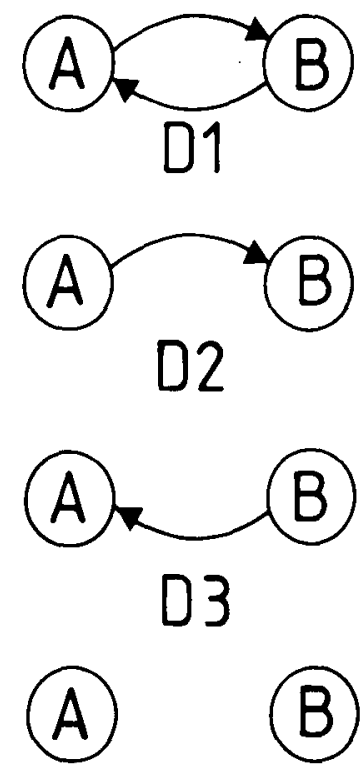

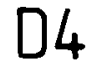

Figure 6. Representations of graphs and digraphs (i.e., directed graphs) in the two-node case.

the first case, pairs of complementary digraph arcs (e.g., the arc from $A$ to $B$, and the arc from $B$ to $A$ ) occur only in conjunction. In the second case, the complementary pairs of arcs occur independently of each other. As a simple example, let the probability of formation of a two-node graph link be $h$. Then, in Figure 6, the Graphs G1 and G2 occur with probabilities $h$ and $(1-\mathrm{h})$, respectively. Hence, the Type (2) and Type $(1,1)$ fragment probabilities-which represent the probabilities of the presence and of the absence, respectively, of an intact path from A to B (or equally from B to A)are also $h$ and $(1-h)$, respectively. In the first digraph interpretation, with conjunctive arcs, the occurrences of Digraphs D1, D2, D3, and D4 have probabilities h, 0, 0, and $(1-h)$, respectively. It is immediately apparent that the probabilities of the presence and the absence of intact paths between $A$ and $B$ are the same as in the graph case, and indeed this first digraph interpretation can be regarded as merely a notational variant of the graph interpretation. The second digraph interpretation, with independent arcs, is of more substantive interest. In this, Digraphs D1, D2, D3, and D4 have probabilities $h^{2}, h(1-h), h(1-h)$, and $(1-h)^{2}$, respectively. Thus, the probability of the presence of an intact path from $A$ to B (or, equally, from B to A) is $h^{2}+h(1-h)$, which equals $h$, whereas the probability of its absence is $h(1-h)+(1-h)^{2}$, which equals $(1-h)$, both values being the same as those provided by the graph interpretation. Similar results hold when the number of nodes takes values greater than two. Only the latter, inde-

pendent-arc type of digraph interpretation is considered further here.

In general, it can be seen that experiments in which a memory is cued on only one occasion do not distinguish the different interpretations of the fragment model. The route from cue (or cues) to target is inherently directional, and thus it makes no difference whether or not the reverse route is intact. But if a memory is probed on more than one occasion, in different directions, the graph interpretation and the independent-arc digraph interpretation of the fragment model yield different predictions. Only the graph interpretation predicts strongly consistent recall. For example, suppose that (reverting to the four-node case) $\mathrm{B}, \mathrm{C}$, and $\mathrm{D}$ are all retrieved on a first cuing by $\mathrm{A}$. Then, a Type (4) fragment is indicated, and the graph interpretation predicts that subsequent cuing of the same memory by B should similarly retrieve A, C, and D. However, according to the digraph interpretation, all that is of relevance that can be concluded from the first cuing is that arcs (or chains of arcs) from $\mathrm{A}$ to $\mathrm{C}$ and from $A$ to $D$ are intact. Thus, at the second cuing, with $B$, it can be predicted only that, if A is recalled, then so should be $\mathrm{C}$ and $\mathrm{D}$. Hence, permitted patterns of recall at the second cuing comprise not only $\mathrm{ACD}$, but also $\mathrm{CD}, \mathrm{C}, \mathrm{D}$, and zero. Two experiments have been reported whose results allow one, in principle, to decide between the graph and the digraph interpretations, but, in practice, neither set of results is conclusive. These studies are described next.

The first of the repeated-cuing experiments was reported by Jones (1978b). However, it investigated the recall of stimuli (photographs of different objects, of different colors, in different locations) whose components each had only a small number of possible values, making the effects of guessing relatively large and difficult to disentangle from the question of consistency in recall. The second relevant experiment is the final one (Experiment 3) reported by Ross and Bower (1981a). In this, subjects were shown clusters of four unrelated words, and subsequently were provided with first one word and then, on a separate occasion, another word as retrieval cues. This was an improvement over the Jones (1978b) experiment because simple guessing was no longer a problem. Ross and Bower examined the fits of the horizontal and schema models to the data from individual cuings separately, and found that the former was the better (this was as would be expected on the basis of the present article, in which the horizontal model is recognized as a constrained version of the fragment model, which is shown to represent the recall of material with unrelated elements well). But the primary point at issue concerns the question of the consistency of recall for the two cuings.

Without describing them in such terms, Ross and Bower (1981a) actually examined the qualitative predictions of the graph and digraph interpretations of the fragment model (these correspond to their fragment and horizontal predictions, respectively). There were 
altogether 864 sequences of recall, which could be classified into 40 patterns. The graph interpretation allows observations in only 9 of these, which turned out to include $747(86 \%)$ of the observed sequences ( 2 of these patterns were those of complete success or complete failure in recall, which together accounted for 522 of the sequences). The digraph interpretation allows a further 16 patterns, which turned out to include 97 of the remaining 117 sequences. This interpretation thus allows, in all, 844 (98\%) of the observed sequences. On the face of it, this result provides support for the weaker digraph restrictions on consistency of recall (although statistical comparisons are not possible because of the low numbers of observations per sequence per subject). However, more detailed examination of the data reveals an artifactual basis for this result.

Let us focus further on the 117 observed sequences that did not conform to the nine patterns allowed by the graph interpretation. Examination of Table 4 of Ross and Bower (1981a) shows that in 13 of these sequences the total numbers of components, irrespective of identity, that were recalled on the first and on the second cuings were the same. For example, there was 1 observed sequence in which $A$ produced recall of $B$ and $\mathrm{C}$, and then B produced recall of $\mathrm{C}$ and D (Pattern 8 of Ross \& Bower, 1981a)-that is, two components were recalled on each cuing. In the great majority-104-of the sequences, however, the total numbers of components recalled on the first and on the second cuings were different. Consider two examples. First, there were 3 observed sequences in which A produced recall of B, $C$, and $D$, and then $B$ produced recall of $C$ and $D$ (Pattern 2)-that is, more components were recalled on the first cuing. Second, there were 17 observed sequences in which $B$ produced recall of $C$ and $D$, and then $A$ produced recall of B, C, and D (Pattern 15)-that is, more components were recalled on the second cuing. Now, what is important is that, overall, the second type of example was very much more frequent than the first type. In 79 of the sequences, there were more components recalled on the second cuing, whereas in only 25 of the sequences were more components recalled on the first cuing. The difference is highly significant $\left[\chi^{2}(1)=28.0\right]$. Thus, most of the observed deviation from the graph interpretation of the fragment model resulted from the recall level's being higher on the second cuing than on the first cuing. But this result is just as unexpected for a digraph model as for a graph model. Both the schema model and the digraph interpretation of the fragment model allow asymmetry in individual sequences of recall, but expect overall incidences of first-cue superiority and second-cue superiority to be equal. Therefore, contrary to the conclusion of Ross and Bower, the results of their repeated-cuing experiment do not provide a basis for choosing between any of the models under consideration. Instead, they point to a completely separate factor-a tendency for subjects to recall more on later trials-which accounts for a relatively small proportion of the variance in that particular experiment.

In summary, the work discussed in this article allows one to conclude that memory for groups of unrelated words is consistent with the fragment rather than with the schema model. The fragment model was interpreted in terms of networks of connected nodes. Patterns of recall were approximately consistent with the link model special case of the fragment model. This predicts the distribution of different types of memories by assuming that each internode connection is encoded independently. It was noted that most experiments cannot distinguish between graph and digraph interpretations of the fragment model, and that thus far those that in principle could do so have not in practice achieved this goal.

\section{REFERENCES}

Abelson, R. P. (1981). Psychological status of the script concept. American Psychologist, 36, 715-729.

ANDERSON, J. R. (1976). Language, memory, and thought. Hillsdale, NJ: Erlbaum.

ANDERson, J. R. (1983). The architecture of cognition. Cambridge, MA: Harvard University Press.

AnDERson, J. R., \& BowER, G. H. (1973). Human associative memory. Washington, DC: Winston.

Anderson, J. R., Kline, P. J., \& Beasley, C. M. (1979). A general learning theory and its application to schema abstraction. In G. H. Bower (Ed.), The psychology of learning and motivation (Vol. 13). New York: Academic Press.

Asch, S. E., \& Ebenholtz, S. M. (1962). The principle of associative symmetry. Proceedings of the American Philosophical Society, 106, 135-163.

BAHrick, H. P. (1970). Two-phase model for prompted recall. Psychological Review, 77, 215-222.

BAHRICK, H. P. (1979). Broader methods and narrower theories for memory research: Comments on the papers by Eysenck and Cermak. In L. S. Cermak \& F. I. M. Craik (Eds.), Levels of processing in human memory. Hillsdale, $\mathrm{NJ}$ : Erlbaum.

Bartlett, F. C. (1932). Remembering. London: Cambridge University Press.

Brondent, D. E. (1981). Association lecture: From the percept to the cognitive structure. In J. Long \& A. Baddeley (Eds.), Attention and performance IX. Hillsdale, NJ: Erlbaum.

Ellis, A. W., \& Marghalt, J. C. (1978). Semantic errors or statistical flukes? A note on Allport's "On knowing the meaning of words we are unable to report." Quarterly Journal of Experimental Psychology, 30, 569-575.

Foss, D. J., \& Harwood, D. A. (1975). Memory for sentences: Implications for human associative memory. Journal of Verbal Learning and Verbal Behavior, 14, 1-16.

Hinton, G. E. (1981). Implementing semantic networks in parallel hardware. In G. E. Hinton \& J. A. Anderson (Eds.), Parallel models of associative memory. Hillsdale, $\mathrm{NJ}$ : Erltaum.

Hollan, J. (1975). Features and semantic memory: Set-theoretic or network model? Psychological Review, 82, 154-155.

Jones, G. V. (1976). A fragmentation hypothesis of memory: Cued recall of pictures and of sequential position. Journal of Experimental Psychology: General, 105, 277-293.

Jones, G. V. (1978a). Recognition failure and dual mechanisms in recall. Psychological Review, 85, 464-469.

Jones, G. V. (1978b). Repeated cuing and the structure of recall. British Journal of Mathematical and Statistical Psychology, 31, $1-10$. 
JoNes, G. V. (1978c). Tests of a structural theory of the memory trace. British Journal of Psychology, 69, 351-367.

Jones, G. V. (1980). Interaction of intrinsic and extrinsic knowledge in sentence recall. In R. S. Nickerson (Ed.), Attention and performance VIII. Hillsdale, NJ: Erlbaum.

Jones, G. V. (1983). Structure of the recall process. Philosophical Transactions of the Royal Society of London (Series B), 302, 373-385. (Reprinted in D. E. Broadbent (Ed.), Functional aspects of human memory. London: The Royal Society, 1983)

Jones, G. V., \& PAYNe, M. S. (1982). Recall and the flexibility of linguistic processing. In A. Flammer \& W. Kintsch (Eds.), Discourse processing. New York: North-Holland.

LE VoI, M. E., \& Rawles, R. E. (1979). Backward recognition failure: A case of retrieval success. Quarterly Journal of Experimental Psychology, 31, 609-620.

Murdock, B. B., JR. (1979). Convolution and correlation in perception and memory. In L.-G. Nilsson (Ed.), Perspectives on memory research. Hillsdale, $\mathrm{NJ}$ : Erlbaum.

Numerical Algorithms Group. (1981). NAG FORTRAN library manual: Mark 9 (Vol. 3). Oxford, England, and Downers Grove, IL: Author.

Paivio, A., Yuille, J. C., \& Madigan, S. A. (1968). Concreteness, imagery, and meaningfulness values for 925 nouns. Journal of Experimental Psychology Monographs, 76(1, Pt. 2).

Richardson, J. T. E. (March 1983). Fragmentation and integration in the retention of complex ideas. Paper presented at the meeting of the Experimental Psychology Society, Manchester, England.

Ross, B. H., \& Bower, G. H. (1981a). Comparisons of models of associative recall. Memory Cognition, 9, 1-16.

Ross, B. H., \& Bowen, G. H. (1981b). Horizontal modelQuartets. Unpublished manuscript.

Rumelhart, D. E., \& Ortony, A. (1977). The representation of knowledge in memory. In R. C. Anderson, R. J. Spiro, \& W. E. Montague (Eds.), Schooling and the acquisition of knowledge. Hillsdale, NJ: Erlbaum.

Thonndike, E. L., \& LoraE, I. (1944). The teacher's word book of 30,000 words. New York: Teacher's College Press, Columbia University.

WICKENS, T. D. (1982). Models for behavior: Stochastic processes in psychology. San Francisco: Freeman.

Wilhite, S. C. (1982). Sentence coding: Tests of the addresscontents model and the fragmentation-conceptual focus hypothesis. Quarterly Journal of Experimental Psychology, 34A, 259-274.

WiLson, R. J. (1972). Introduction to graph theory. Edinburgh, Scotland: Oliver \& Boyd.

\section{NOTES}

1. The number of nonisomorphic structures increases rapidly with the number of nodes. If the latter is doubled from 4 to 8 , the former increases more than a thousandfold, from 11 to 12,346 (see Wilson, 1972, p. 162).

2. Ross and Bower (1981a) effectively extracted predictions from a digraph rather than a graph interpretation of the horizontal model. As shown later in this article, the two interpretations are equivalent in the type of experiment under consideration. Nevertheless, the algebraic form in which the Ross and Bower predictions are cast [see Ross \& Bower, 1981a (pp. 2-3), $1981 \mathrm{~b}$ ] is not obviously equivalent to those developed here. But I have checked that the two sets of equations yield numerically identical predictions.
3. It is readily shown that, for example, the link version of the fragment model can be written as a special case of the general version. Redefine the four independent parameters of the general version as $\theta, \alpha, \beta$, and $\gamma$, where $\theta$ is a link probability that gives rise to a set of fragment probabilities, $\mathrm{fL}_{(\mathrm{i})}$, as described earlier (Equations 8 to 13 ), and $\alpha, \beta$, and $\gamma$ may each take values between -1 and 1 inclusive. Then, it is clear that the unconstrained general version may be characterized via fragment probabilities defined as $f_{(4)}^{G}=f_{(4)} f_{(4)} f_{(3,1)}=f_{(3,1)}+\alpha$, $\mathrm{fG}_{(2,1,1)}=\mathrm{fL}_{(2,1,1)}+\beta, \mathrm{fG}_{(2,2)}=\mathrm{fL}_{(2,2)}+\gamma$, and $\mathrm{fG}_{(1,1,1,1)}$ $=1-f^{G}(4)-f_{(3,1)}-f_{(2,1,1)}-f^{G} G_{(2,2)}$. It is clear also that the link version corresponds to the special case of the general version for which $\alpha=\beta=\gamma=0$. Note further, however, that if it were possible to distinguish experimentally each of the 11 different nonisomorphic structures shown in Figure 2 (rather than simply the five types of fragment into which they can be grouped on the basis of their numbers of interconnected nodes), the general model would then in principle require 10 rather than 4 independent parameters.

4. Since an absolute test of the goodness of fit of a model will in general indicate a significant discrepancy with data whenever sampling of the latter is continued sufficiently extensively, it can be argued (e.g., Wickens, 1982, chap. 6) that comparative tests between two or more models are in principle more informative than absolute tests.

\section{APPENDIX}

It is shown here that the schema model is not a special case of the fragment model. Some general relations among data that are predicted by the fragment model are contradicted by the schema model. In the four-word case, for example, the fragment model predicts that the probability of recalling both words to a two-word cue cannot exceed the probability of recalling at least one word to a one-word cue, whereas the schema model predicts that it may, as follows. Denote the difference between these two probabilities by $d$. That is,

$$
d=P(1 \mid 1)+P(2 \mid 1)+P(3 \mid 1)-P(2 \mid 2) .
$$

Then, according to the fragment model,

$$
\begin{aligned}
d= & (1 / 2) f_{(2,1,1)}+f_{(2,2)}+(3 / 4) f_{(3,1)}+f_{(4)} \\
& -\left[f_{(4)}+(1 / 2) f_{(3,1)}+(2 / 3) f_{(2,2)}\right] \\
= & (1 / 4) f_{(3,1)}+(1 / 3) f_{(2,2)}+(1 / 2) f_{(2,1,1)}
\end{aligned}
$$

which is necessarily greater than or equal to zero. But according to the schema model,

$$
d=3 a r(1-r)^{2}+3 a r^{2}(1-r)+a r^{3}-a(2-a) r^{2},
$$

which, in contrast, may take negative values (e.g., for $r=1$ and $\mathrm{a}=1 / 2, \mathrm{~d}=-1 / 4)$.

(Manuscript received May 4, 1983; revision accepted for publication January 5,1984 .) 